\title{
Performance Comparison of Total Variation based Image Regularization Algorithms
}

\author{
V Kamalaveni $^{\# 1}$, K A Narayanankutty ${ }^{*}$, S Veni $^{2}$ \\ \# Department of Computer Science and Engineering, Amrita School of Engineering, Coimbatore, \\ Amrita Vishwa Vidyapeetham, Amrita University, India \\ * Amrita School of Engineering, Coimbatore, Amrita Vishwa Vidyapeetham, Amrita University, India \\ E-mail:ka_narayanankutty@yahoo.com \\ ${ }^{2}$ Department of Electronics and Communication Engineering, Amrita School of Engineering, Coimbatore, \\ Amrita Vishwa Vidyapeetham, Amrita University, India \\ E-mail: s_veni@cb.amrita.edu \\ ${ }^{1}$ Corresponding author: Tel.+91-956-661-8469 E-mail: v_kamalaveni@cb.amrita.edu
}

\begin{abstract}
The mathematical approach calculus of variation is commonly used to find an unknown function that minimizes or maximizes the functional. Retrieving the original image from the degraded one, such problems are called inverse problems. The most basic example for inverse problem is image denoising. Variational methods are formulated as optimization problems and provides a good solution to image denoising. Three such variational methods Tikhonov model, ROF model and Total Variation-L1 model for image denoising are studied and implemented. Performance of these variational algorithms are analyzed for different values of regularization parameter. It is found that small value of regularization parameter causes better noise removal whereas large value of regularization parameter preserves well sharp edges. The Euler's Lagrangian equation corresponding to an energy functional used in variational methods is solved using gradient descent method and the resulting partial differential equation is solved using Euler's forward finite difference method. The quality metrics are computed and the results are compared in this paper.
\end{abstract}

Keywords - total variation; Calculus of variation; Functional; gradient descent method; fidelity term

\section{INTRODUCTION}

Variational models have been sucessfully used for image denoising and remains an active area of research. In variational method it is required to define cost or energy functional which characterizes the image structure to be enhanced. The variational technique also involves the minimization of energy functional which can be implemented using calculus of variation, leading to a partial differential equation. The calculus of variation is concerned with functional which are functions whose arguments are functions or whose domain is functions and the range is a real number. Several variational regularization models have been proposed by the researchers. Tikhonov[1] proposed regularization functional which is smoothing edges excessively, so the edges are not preserved properly. To resolve this problem Rudin et al.[2] developed a new total variation based denoising method. Junfeng Yang et al. used
12 TV-norm [4]. Y.Wang et al. used 11-norm as regularizer[10]. The 10-norm is used as regularizing term in Potts model [16]. The authors Haijuan $\mathrm{Hu}$ et al. used nonlocal total variation in their work[7].

Different data fidelity terms are used in variational models. The ROF model [2] making use of the squared 12 fidelity term is efficient in removing gaussian noise. Christian Clason et al. [5] and Junfeng Yang et al.[4] in their works used 11-norm as data fidelity term. It is found that 102-norm as data-fidelity term shown good performance in eliminating impulse and gaussian noise [11]. To remove the impulse noise present in the images the 10-norm is used as data fidelity term [9] and infinity norm is used for uniform noise [6]. Manya $\mathrm{V}$ Afonse et al. used weighted TV-norm in year 2015[18]. Kui-Liu et al. used H-1-norm as data fidelity[20]. Mazlinda Ibrahim et al. used Gaussian curvature as regulariser[21]. Yao Zhao et al. proposed 
Adaptive TV based regularization algorithm for synthetic aperture radar image despeckling [19].

Our paper is written as follows. In section 1, introduction of the topic is given. In section 2, fundamental concepts in Calculus of Variation is discussed. In section 3, solving a variational problem using Euler Lagrangian equation is detailed. The variational methods ROF model, Tikhonov model and TV-L1 are discussed in chapters four, five and six respectively. Performance of variational methods is discussed with numerical results in section 7 and the last section concludes the paper.

\section{CAlculus OF VARIATION}

The calculus of variation deals with the maxima or minima of functionals, known as extrema. Determining the extrema of functionals is similar to finding minima and maxima of functions. The minima and maxima of a function can be found by finding the points where its derivative becomes equal to zero. The extrema of functionals can be found by finding functions where the derivative of functional is equal to zero. This gives us the Euler-Lagrange equation of the functional.

Since a functional maps a function to a number, the functional usually makes use of integral.

$$
P[t(x)]=\int_{0}^{\pi}[t(x)]^{2} d x
$$

is a functional. When $t(x)=x$ then the functional value is $\pi^{3}$

3

Another example of functional which are used in the calculus of variation is given below.

$$
P[t(x)]=\int_{x 1}^{x 2} F\left(x, t(x), t^{\prime}(x)\right) d x
$$

Where $t^{\prime}(x)=\frac{d t(x)}{d x}$

Several engineering problems can be modeled as variational problems and these problems can be solved by finding the function that minimizes or maximizes the functional. Thus a variational problem becomes an optimization problem.

\section{EULER LAGRANGIAN EQUATION}

The functional with higher order derivatives of $t(x)$ is below.

$$
P[t(x)]=\int_{x 1}^{x 2} F\left(x, t(x), t^{\prime}(x), t^{\prime \prime}(x)\right) d x
$$

The unknown function that minimizes or maximizes $\mathrm{P}[\mathrm{t}(\mathrm{x})]$ should satisfy the following Euler-Lagrange equation.

$$
\frac{\partial F}{\partial t}-\frac{d}{d x} \frac{\partial F}{\partial t^{\prime}}+\frac{d^{2}}{d x^{2}} \frac{\partial F}{\partial t^{\prime \prime}}=0
$$

The functional with $\mathrm{i}^{\text {th }}$ derivative of $\mathrm{t}$, the Euer-Lagrange equation is

$$
\sum_{i=0}^{n}(-1)^{i} \frac{d^{i}}{d x^{i}} \frac{\partial F}{\partial t^{(i)}}=0
$$

In image processing applications we need find the required image $\mathrm{t}$ as a function of $\mathrm{i}$ and $\mathrm{j}$, satisfying the following functional.

$$
\min _{t} P[t(i, j)]=\min _{t} \int_{a}^{b} F\left(i, j, t(i, j), t_{i}(i, j), t_{j}(i, j)\right) d i d j
$$

Euler lagrangian equation corresponding to this functional is

$$
\frac{\partial F}{\partial t}-\frac{\partial}{\partial i} \frac{\partial F}{\partial t_{i}}-\frac{\partial}{\partial j} \frac{\partial F}{\partial t_{j}}=0
$$

A different approach for denoising is to suppress uncorrelated random intensity variations in an image. Mathematical definition of total variation (TV) is a quantity measuring ups and downs in the function values. The noise present in an image causes an increase in the total variation. So the denoising can be implemented by minimizing the total variation corresponding to an image. The anisotropic TV-norm of the 1-D digital signal(f) is defined as [3][17].

$$
T V(f)=\sum_{i=2}^{N}\left|f_{i}-f_{i-1}\right|
$$

Here $f_{i}$ and $f_{i-1}$ are the value of function at $i^{\text {th }}$ and $i-1^{\text {th }}$ instant of time. For a continuous function(f) the total variation norm is defined as

$$
T V(f)=\int_{x}\left|f^{\prime}(x)\right| d x
$$

This extends to 2-D by looking at the total jump horizontally and vertically. For denoising an image $\mathrm{u}(\mathrm{x}, \mathrm{y})$, we want to minimize the TV-norm defined as[15].

$$
T \text { Vnorm }=T V(u)=\int_{\Omega}|\nabla u| d \Omega
$$

Here $\nabla u$ is the gradient of the image. In addition the restored image $u$ to resemble original noisy image $u 0$, the second term matching or fidelity term is defined as follows.

$$
\text { fidelityterm }=\int_{\Omega}\left|\left(u-u_{0}\right)\right|^{2} d \Omega
$$

We should lower both TV-norm and fidelty term for denoising. However fully lowering TV-norm will result in totally blurred image and fully lowering fidelity term gives noisy image. For image denoising what we need is a compromise solution which is formulated as finding a function $\mathrm{u}$ which minimizes the following functional given in eqn.11. This is the core idea used in the variational methods like ROF model, Tikhonov model and TV-L1 models.

$$
\min _{u} \int_{\Omega}|\nabla u| d \Omega+\int_{\Omega}\left(u-u_{0}\right)^{2} d \Omega
$$




\section{ROF MODEL}

ROF model is a strict convex optimization method and it has unique global minimizer [2][8]. Here numerical algorithm for finding minimizer of ROF model is presented. The ROF model is described as a constrained optimization problem given in equation 12 .

$$
\min _{u}\{E(u)\}=\int_{\Omega}|\nabla u(x, y)| d \Omega+\lambda \int_{\Omega}\left(u-u_{0}\right)^{2} d \Omega
$$

Here $\mathrm{u}_{0}$ is given noisy image, $\mathrm{u}$ is denoised image and $\lambda$ is a regularization parameter, a constant that decides the tradeoff between two terms.

The denoised image $\mathrm{u}$ is found using following procedure [12]. Step-1. Finding Euler-lagrangian equation for the above functional. Step-2. Solving Euler-lagrangian equation using gradient descent method and obtain the solution as a partial differential equation. Step-3. Solving the partial differential equation using Euler forward method and obtain the solution $\mathrm{u}$.

Here

$$
F=\lambda\left(u-u_{0}\right)^{2}+\sqrt{u_{x}^{2}+u_{y}^{2}}
$$

Euler Langrange Equation is given by

$$
\begin{gathered}
\frac{\partial F}{\partial u}-\frac{\partial}{\partial x} \frac{\partial F}{\partial u_{x}}+\frac{\partial}{\partial y} \frac{\partial F}{\partial u_{y}}=0 \\
\frac{\partial}{\partial x} \frac{\partial F}{\partial u_{x}}=\frac{u_{x x} u_{y}^{2}-u_{x} u_{y} u_{x y}}{\left(u_{x}^{2}+u_{y}^{2}\right)^{3 / 2}} \\
\frac{\partial}{\partial y} \frac{\partial F}{\partial u_{y}}=\frac{u_{y y} u_{x}^{2}-u_{x} u_{y} u_{x y}}{\left(u_{x}^{2}+u_{y}^{2}\right)^{3 / 2}}
\end{gathered}
$$

The resulting Euler Lagrangian equation is given by

$$
2 \lambda\left(u-u_{0}\right)-\frac{u_{x x} u_{y}^{2}-2 u_{x} u_{y} u_{x y}+u_{y y} u_{x}^{2}}{\left(u_{x}^{2}+u_{y}^{2}\right)^{3 / 2}}=0
$$

Solving Euler Lagrangian equation using the gradientdescent method [13], we obtain

$$
\frac{\partial u}{\partial t}=-\frac{\partial E}{\partial u}=\frac{u_{x x} u_{y}^{2}-2 u_{x} u_{y} u_{x y}+u_{y y} u_{x}^{2}}{\left(u_{x}^{2}+u_{y}^{2}\right)^{3 / 2}}-2 \lambda\left(u-u_{0}\right)
$$

Using Euler's forward method the above equation is solved as follows.

$$
\begin{aligned}
& \left(u_{x x}\right)_{i, j}^{n}=u_{i+1, j}^{n}+u_{i-1, j}^{n}-2 u_{i, j}^{n} \\
& \left(u_{y y}\right)_{i, j}^{n}=u_{i, j+1}^{n}+u_{i, j-1}^{n}-2 u_{i, j}^{n}
\end{aligned}
$$

$$
\begin{aligned}
& \left(u_{x y}\right)_{i, j}^{n}=\frac{1}{4}\left(u_{i+1, j+1}^{n}+u_{i-1, j-1}^{n}-u_{i-1, j+1}^{n}-u_{i+1, j-1}^{n}\right) \\
& \left(u_{x}\right)_{i, j}^{n}=\frac{u_{i+1, j}^{n}-u_{i-1, j}^{n}}{2} \\
& \left(u_{y}\right)_{i, j}^{n}=\frac{u_{i, j+1}^{n}-u_{i, j-1}^{n}}{2} \\
& \text { Num }=\left(\left(u_{x x}\right)_{i, j}^{n}\left(\left(u_{y}\right)_{i, j}^{n}\right)^{n}-2\left(u_{x}\right)_{i, j}^{n}\left(u_{y}\right)_{i, j}^{n}\left(u_{x y}\right)_{i, j}^{n}+\left(u_{y y}\right)_{i, j}^{n}\left(\left(u_{x}\right)_{i, j}^{n}\right)^{2}\right) \\
& \operatorname{Den}=\left(\left(\left(u_{x}\right)_{i, j}^{n}\right)^{2}+\left(\left(u_{y}\right)_{i, j}^{n}\right)^{2}\right)^{3 / 2}+0.01 \\
& u_{i, j}^{n}=u_{i, j}^{n}+\Delta t\left(\frac{N u m .}{\operatorname{Den} .}-\lambda\left(u_{i, j}^{n}-u_{0}\right)\right)
\end{aligned}
$$

\section{TIKHONOV MODEL}

The commonly used regularization method is Tikhonov model. In this model regularizer term uses the square of the gradient and the fidelity term is $1_{2}$ residual norm [15]. The equation 18 desc ribes this model.

$$
\min _{u}\{E(u)\}=\int_{\Omega}|\nabla u(x, y)|^{2} d \Omega+\lambda \int_{\Omega}\left(u-u_{0}\right)^{2} d \Omega
$$

Here

$$
F=\lambda\left(u-u_{0}\right)^{2}+\left(u_{x}^{2}+u_{y}^{2}\right)
$$

Euler's Equation of functional $\mathrm{F}$ is

$$
\begin{gathered}
\frac{\partial F}{\partial u}-\frac{\partial}{\partial x} \frac{\partial F}{\partial u_{x}}+\frac{\partial}{\partial y} \frac{\partial F}{\partial u_{y}}=0 \\
\frac{\partial}{\partial x} \frac{\partial F}{\partial u_{x}}=2 u_{x x} \quad \frac{\partial}{\partial y} \frac{\partial F}{\partial u_{y}}=2 u_{y y}
\end{gathered}
$$

The resulting Euler Lagrangian equation is below.

$$
\lambda\left(u-u_{0}\right)-\left(u_{x x}+u_{y y}\right)=0
$$

Solving Euler Lagrangian equation by making use of the gradient-descent method, we obtain

$$
\frac{\partial u}{\partial t}=-\frac{\partial E}{\partial u}=\left(u_{x x}+u_{y y}\right)-\lambda\left(u-u_{0}\right)
$$

Using Euler's forward method the above equation is solved as follows.

$$
u_{i, j}^{n+1}=u_{i, j}^{n}+\Delta t\left[\left(u_{x x}\right)_{i, j}^{n}+\left(u_{y y}\right)_{i, j}^{n}-\lambda\left(u_{i, j}^{n}-u_{0}\right)\right]
$$




\section{TV-L1 MODEL}

TV-L1 is not a strict convex optimization method and the global minimizer is not unique. TV-L1 model like ROF model uses same regularizing term but it uses $1_{1}$-norm in fidelity term and its equation is 21 .

$$
\min _{u}\{E(u)\}=\int_{\Omega}|\nabla u(x, y)| d \Omega+\lambda \int_{\Omega}\left|u-u_{0}\right| d \Omega
$$

The lagrangian equation of TV-L1 model is below.

$$
\lambda \frac{\left(u-u_{0}\right)}{\left|u-u_{0}\right|}-\frac{u_{x x} u_{y}^{2}-2 u_{x} u_{y} u_{x y}+u_{y y} u_{x}^{2}}{\left(u_{x}^{2}+u_{y}^{2}\right)^{3 / 2}}=0
$$

Solving Euler Lagrangian equation using the gradientdescent method, we obtain

$$
\frac{\partial u}{\partial t}=-\frac{\partial E}{\partial u}=\frac{u_{x x} u_{y}^{2}-2 u_{x} u_{y} u_{x y}+u_{y y} u_{x}^{2}}{\left(u_{x}^{2}+u_{y}^{2}\right)^{3 / 3}}-\lambda \frac{\left(u-u_{0}\right)}{\left|u-u_{0}\right|}
$$

Using Euler's forward method the above equation is solved as follows.

Num $=\left(\left(u_{x x}\right)_{i, j}^{n}\left(\left(u_{y}\right)_{i, j}^{n}\right)^{2}-2\left(u_{x}\right)_{i, j}^{n}\left(u_{y}\right)_{i, j}^{2}\left(u_{x y}\right)_{i, j}^{n}+\left(u_{y y}\right)_{i, j}^{n}\left(\left(u_{x}\right)_{i, j}^{n}\right)^{2}\right)$

Den $=\left(\left(\left(u_{x}\right)_{i, j}^{n}\right)^{2}+\left(\left(u_{y}\right)_{i, j}^{n}\right)^{2}\right)^{3 / 2}+0.01$

$u_{i, j}^{n}=u_{i, j}^{n}+\Delta t\left(\frac{N u m .}{\text { Den. }}-\lambda \frac{\left(u_{i, j}^{n}-u_{0}\right)}{\left|u_{i, j}^{n}-u_{0}\right|}\right)$

TV-L1 is efficient in removing impulse noise compare to ROF Model. TV-L1 method has the ability to preserve contrast. Contrast invariance means if $\mathrm{u}(\mathrm{x}, \mathrm{y})$ is the denoised image corresponding to noisy image $\mathrm{u}_{0}$, then $\mathrm{cu}(\mathrm{x}, \mathrm{y})$ is the denoised image of noisy image $\mathrm{cu}_{0}$.

\section{EXPERIMENTAL RESULTS}

Salt and pepper noise having density $\mathrm{d}=0.01$ is added synthetically and then images are denoised using variational models i.e ROF model, Tikhonov model and TV-L1 model. The quality of denoised images generated by different variational models are compared which is shown in Fig 1-12 and Table.I-IV shows the summary of PSNR \& SSIM values computed for these methods for different values of regularization parameter i.e $\lambda=0.003,0.01,0.1$ and 0.2 . The quality metrics PSNR and SSIM are defined[14] using equations (23) and (24).

$$
P S N R=10 \log _{10}\left(\frac{255^{2}}{\frac{1}{M N} \sum_{x=0}^{M-1} \sum_{y=0}^{N-1}\left[u(x, y)-u_{0}(x, y)\right]^{2}}\right)
$$

here $\mathrm{u}$ and $\mathrm{u}_{0}$ are denoised image and noisy image and $\mathrm{M}: \mathrm{N}$ represents the number of pixels along the horizontal and vertical direction.
$\operatorname{SSIM}(u, u 0)=\frac{\left(2 \mu_{u} \mu_{u 0}+c_{1}\right)\left({ }^{2} \sigma_{u u 0}+c_{2}\right)}{\left(\mu_{u}^{2}+\mu_{u 0}^{2}+c_{1}\right)\left(\sigma_{u}^{2}+\sigma_{u 0}^{2}+c_{2}\right)}$

$\mu_{\mathrm{u}}$ and $\sigma_{\mathrm{u}}{ }^{2}$ are mean and variance of $\mathrm{u}$ respectively. The covariance of images $\mathrm{u}$ and $\mathrm{u}_{0}$ is is $\sigma_{\mathrm{uu} 0} . \mathrm{C}_{1}$ and $\mathrm{C}_{2}$ are two constants.

In Tables I, II, III and IV we notice that PSNRs and SSIMs of the denoised images in Tikhonov model are lower than those in the ROF model and TV-L1. Another observation from the Tables I, II, III and IV is that SSIM value remains same for TV-L1 model for $\lambda=0.003,0.001,0.1$ and 0.2. Third observation from Tables I-IV is that increases in $\lambda$ causes an increase in SSIM and PSNR values of ROF model and Tikhonov model. In Addition Table III and IV, the increase in SSIM and PSNR values is very obvious for ROF model.

Figs.1-4 is for results of fruits image and Figs. 5-8 is for results of cameraman image. Fig.1-2 and Fig. 5-6 for $\lambda=0.003$ and 0.01 , Tikhonov model smoothing is more and edges are not preserved whereas ROF model and TV-L1 is able to preserve edges. Fig.3-4 and Fig.7-8 for $\lambda=0.1$ and 0.2 Tikhonov model amount of smoothing is less and is able to preserve strong edges and the ROF model preserves very well sharp edges and fine details compare to TV-L1 and Tikhonov model. In Fig. $1-8$ for $\lambda=0.003,0.01,0.1,0.2$ visual quality of the restored image generated by TV-L1 model is same. This means that contrast is invariant also noise removing and edge preserving effect is same for $\lambda=$ 0.003,0.01,0.1,0.2 in TV-L1 method.

TABLE I

PSNR AND SSIM FOR ROF MODEL, TV-L1, TIKHONOV $\Lambda=0.003$

\begin{tabular}{|c|l|l|l|l|l|l|}
\hline Image & ROF & $\begin{array}{l}\text { TV- } \\
\text { L1 }\end{array}$ & TIKho & ROF & $\begin{array}{l}\text { TV- } \\
\text { L1 }\end{array}$ & Tikho \\
\hline & SSIM & SSIM & SSIM & PSNR & PSNR & PSNR \\
\hline lena & 0.65 & 0.74 & 0.41 & 24.31 & 25.28 & 18.66 \\
\hline fruits & 0.71 & 0.70 & 0.45 & 23.96 & 24.50 & 18.59 \\
\hline camera & 0.72 & 0.70 & 0.41 & 24.81 & 24.39 & 13.88 \\
\hline coins & 0.73 & 0.71 & 0.39 & 23.73 & 23.20 & 16.62 \\
\hline ship & 0.73 & 0.72 & 0.44 & 25.05 & 22.63 & 18.36 \\
\hline lift & 0.65 & 0.65 & 0.56 & 25.82 & 25.82 & 23.31 \\
\hline
\end{tabular}

TABLE II

PSNR AND SSIM FOR ROF MODEL, TV-L1, TIKHONOV $\lambda=0.01$

\begin{tabular}{|c|l|l|l|l|l|l|}
\hline Image & ROF & $\begin{array}{l}\text { TV- } \\
\text { L1 }\end{array}$ & TIKho & ROF & $\begin{array}{l}\text { TV- } \\
\text { L1 }\end{array}$ & Tikho \\
\hline & SSIM & SSIM & SSIM & PSNR & PSNR & PSNR \\
\hline lena & 0.76 & 0.74 & 0.46 & 25.15 & 23.93 & 18.68 \\
\hline fruits & 0.72 & 0.70 & 0.46 & 24.69 & 24.89 & 18.76 \\
\hline camera & 0.72 & 0.70 & 0.43 & 24.96 & 23.67 & 13.90 \\
\hline coins & 0.74 & 0.72 & 0.42 & 23.96 & 22.92 & 16.84 \\
\hline ship & 0.75 & 0.74 & 0.47 & 25.94 & 24.73 & 18.55 \\
\hline lift & 0.67 & 0.65 & 0.57 & 26.07 & 25.90 & 23.48 \\
\hline
\end{tabular}




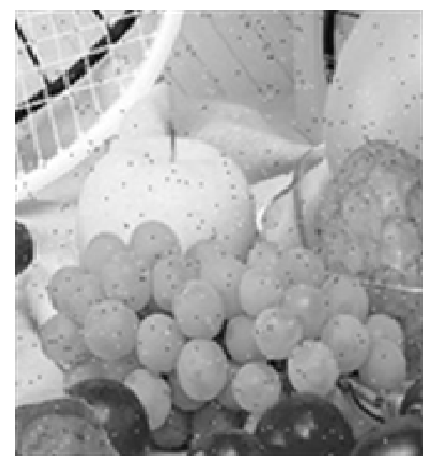

a

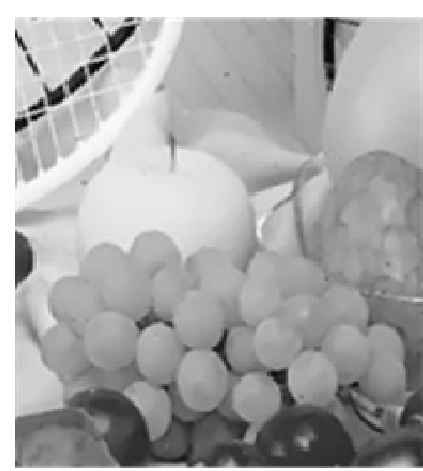

18.

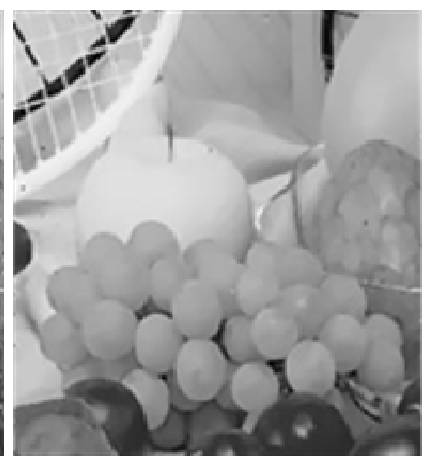

lit

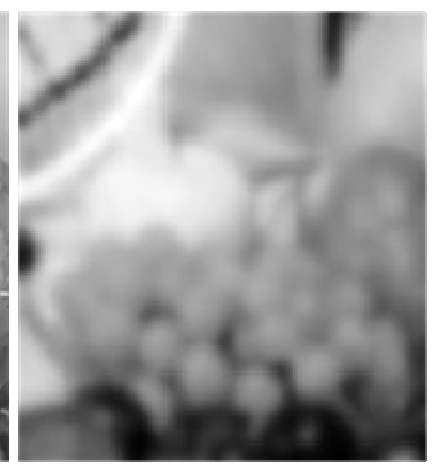

d d

Fig 1. (a) noisy image (b)denoised image ROF model $\lambda=0.003$ (b) denoised image TV-L1 model $\lambda=0.003 \quad$ (d) denoised image Tikhonov model $\lambda=0.003$

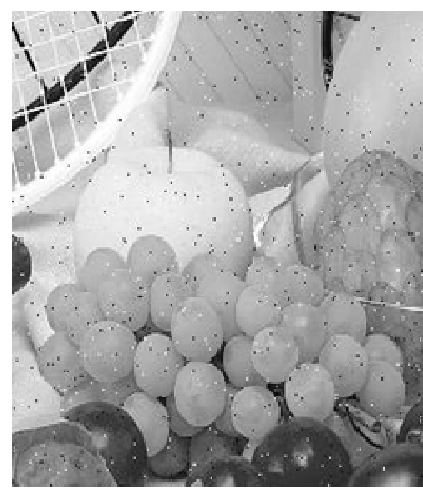

(a)

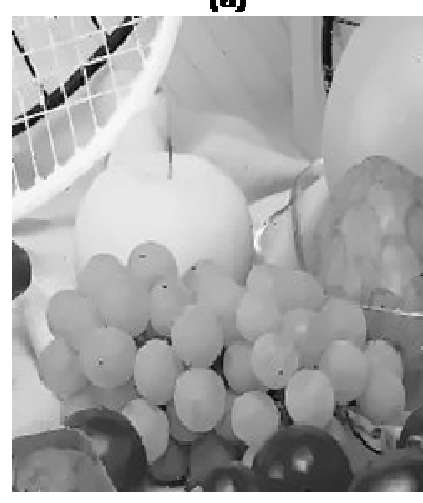

(c)

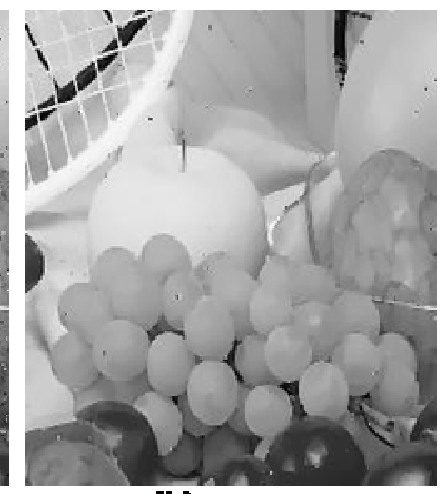

(b)

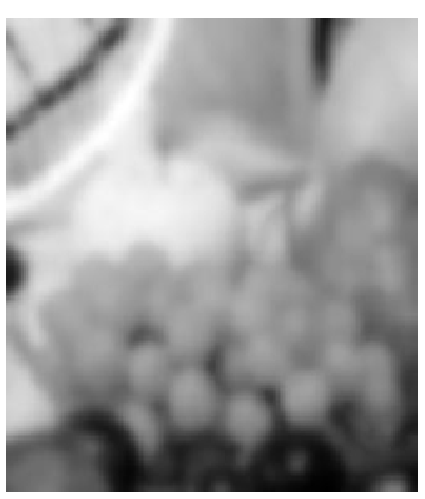

(d)

Fig.2. (a) noisy image (b) denoised image ROF model $\lambda=0.01$ (b) denoised image TV-L1 model $\lambda=0.01 \quad$ (d) denoised image Tikhonov model $\lambda=0.01$

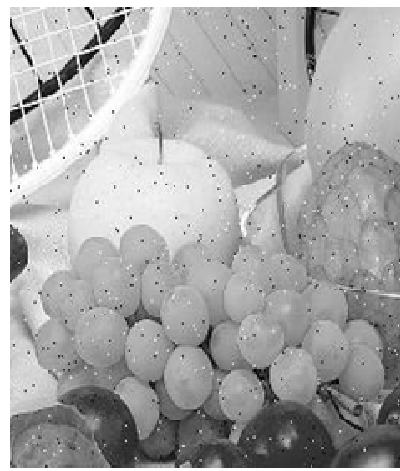

(a)

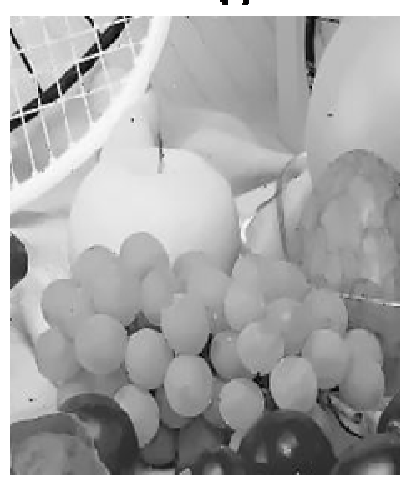

(c)

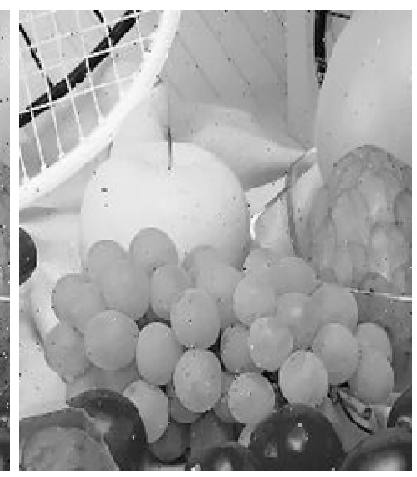

(b)

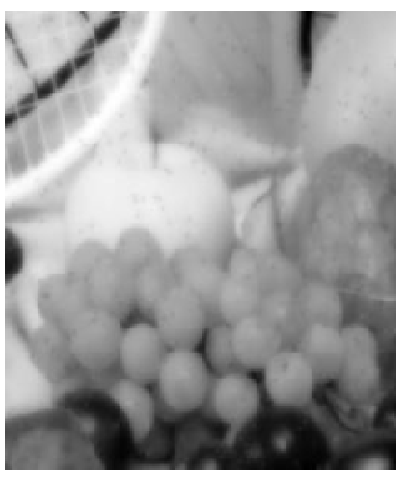

(d)
Fig.3. (a) noisy image (b) denoised image ROF model $\lambda=0.1$ (b) denoised image TV-L1 model $\lambda=0.1 \quad$ (d) denoised image Tikhonov model $\lambda=0.1$
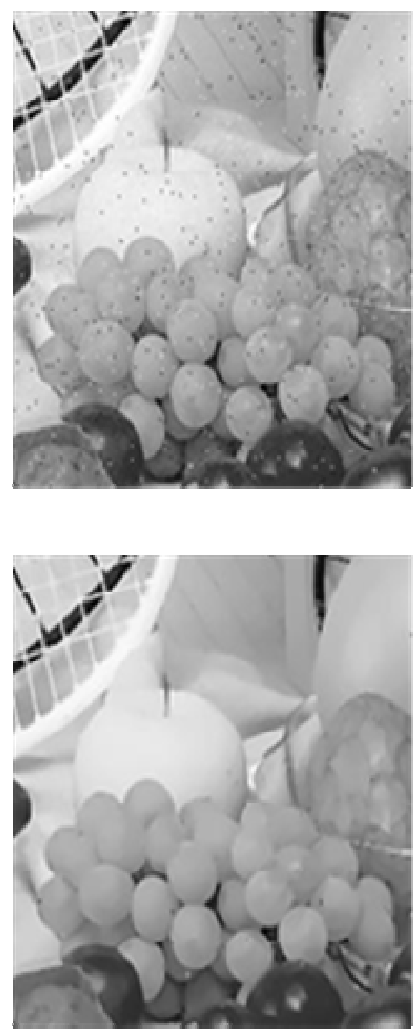

"I!

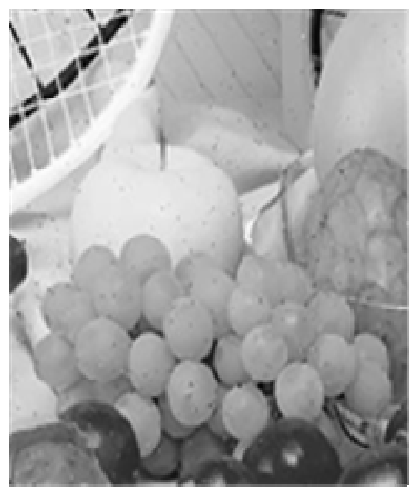

it...

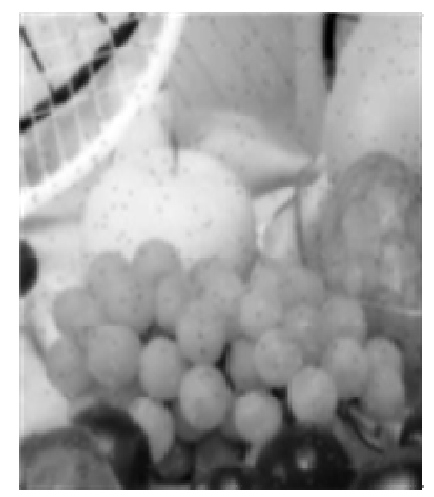

adi
Fig.4. (a) noisy image (b) denoised image ROF model $\lambda=0.2$ (b) denoised image TV-L1 model $\lambda=0.2 \quad$ (d) denoised image Tikhonov model $\lambda=0.2$ 


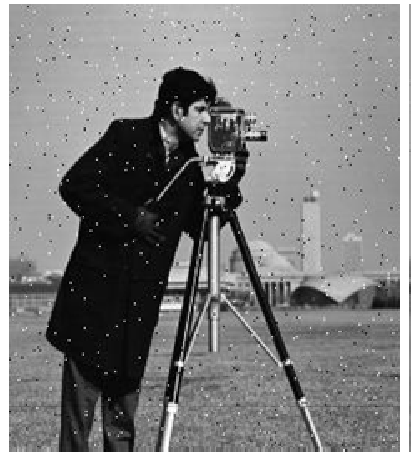

(a)

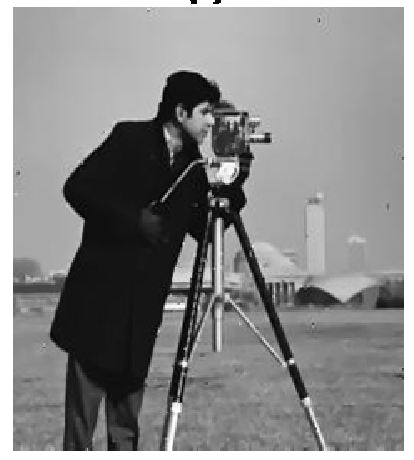

(c)

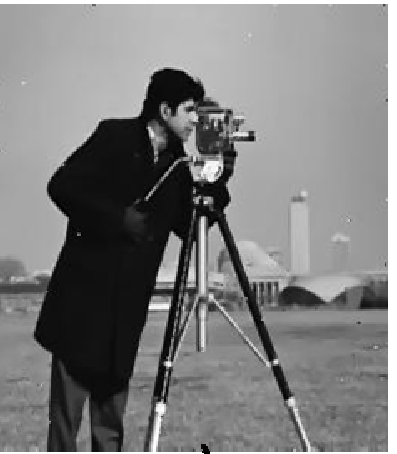

(b)

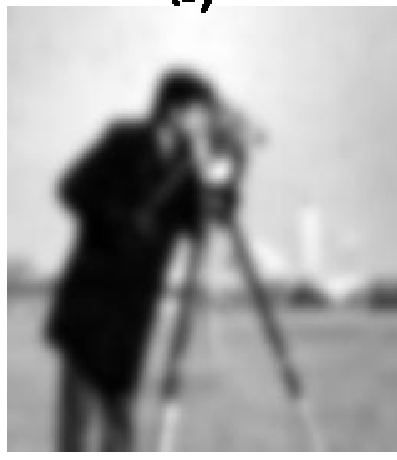

(d)
Fig 5. (a) noisy image (b)denoised image ROF model $\lambda=0.003$ (b) denoised image TV-L1 model $\lambda=0.003 \quad$ (d) denoised image Tikhonov model $\lambda=0.003$

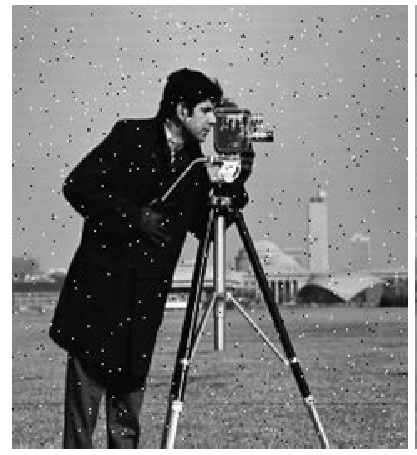

(a)

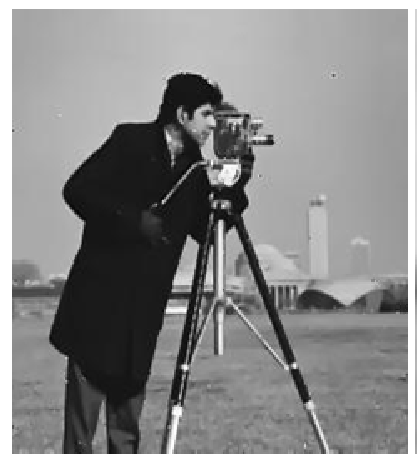

(c)

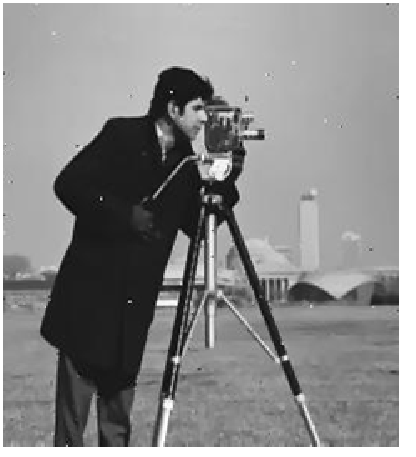

(b)

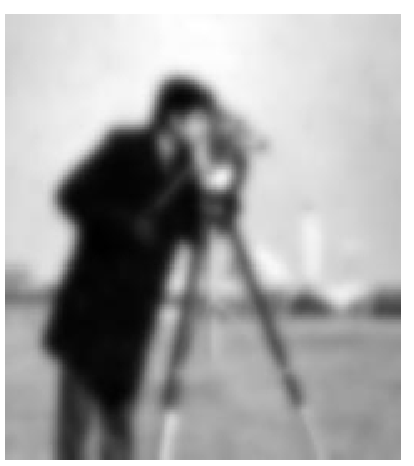

(d)
Fig 6. (a) noisy image (b)denoised image ROF model $\lambda=0.01$ (b) denoised image TV-L1 model $\lambda=0.01 \quad$ (d) denoised image Tikhonov model $\lambda=0.01$

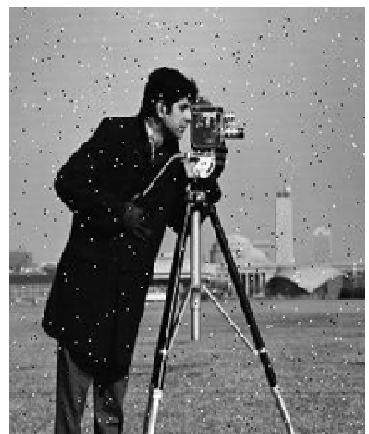

(a)

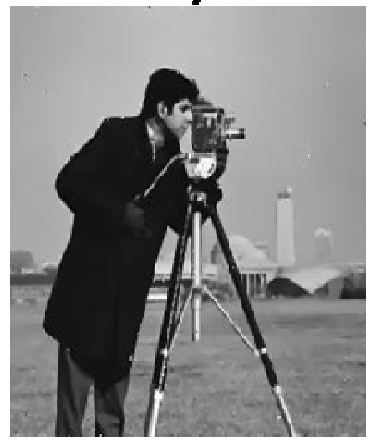

[c]

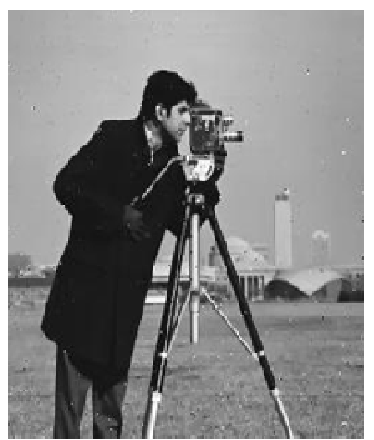

(b]

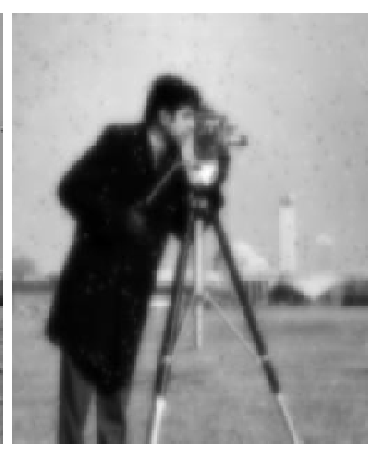

(d)
Fig 7. (a) noisy image (b)denoised image ROF model $\lambda=0.1$ (b) denoised image TV-L1 model $\lambda=0.1 \quad$ (d) denoised image Tikhonov model $\lambda=0.1$
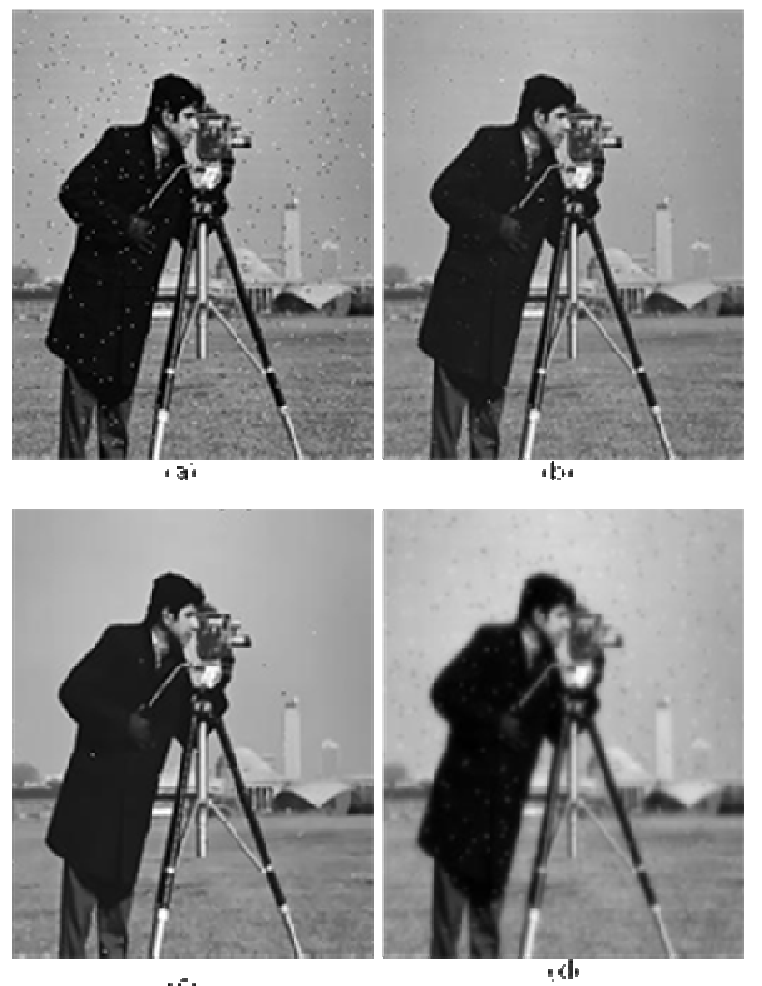

Fig 8. (a) noisy image (b) denoised image ROF model $\lambda=0.2$ (b) denoised image TV-L1 model $\lambda=0.2 \quad$ (d) denoised image Tikhonov model $\lambda=0.2$ 
TABLE III

PSNR AND SSIM FOR ROF MODEL, TV-L1, TIKHONOV $\lambda=0.1$

\begin{tabular}{|c|l|l|l|l|l|l|}
\hline Image & ROF & $\begin{array}{l}\text { TV- } \\
\text { L1 }\end{array}$ & TIKho & ROF & $\begin{array}{l}\text { TV- } \\
\text { L1 }\end{array}$ & Tikho \\
\hline & SSIM & SSIM & SSIM & PSNR & PSNR & PSNR \\
\hline lena & 0.87 & 0.74 & 0.61 & 28.26 & 24.88 & 19.85 \\
\hline fruits & 0.83 & 0.69 & 0.60 & 26.57 & 24.70 & 19.72 \\
\hline camera & 0.78 & 0.70 & 0.57 & 24.92 & 24.17 & 17.58 \\
\hline coins & 0.80 & 0.70 & 0.54 & 25.08 & 21.25 & 17.39 \\
\hline ship & 0.84 & 0.73 & 0.62 & 26.89 & 23.54 & 19.55 \\
\hline lift & 0.76 & 0.64 & 0.65 & 27.61 & 25.72 & 24.85 \\
\hline
\end{tabular}

TABLE IV

PSNR AND SSIM FOR ROF MODEL, TV-L1, TIKHONOV $\lambda=0.2$

\begin{tabular}{|c|l|l|l|l|l|l|}
\hline Image & ROF & $\begin{array}{l}\text { TV- } \\
\text { L1 }\end{array}$ & TIKho & ROF & $\begin{array}{l}\text { TV- } \\
\text { L1 }\end{array}$ & Tikho \\
\hline & SSIM & SSIM & SSIM & PSNR & PSNR & PSNR \\
\hline lena & 0.91 & 0.75 & 0.70 & 30.26 & 24.57 & 21.55 \\
\hline fruits & 0.89 & 0.69 & 0.66 & 28.68 & 24.69 & 21.02 \\
\hline Camera & 0.83 & 0.69 & 0.65 & 26.85 & 23.61 & 20.02 \\
\hline coins & 0.85 & 0.72 & 0.63 & 27.01 & 22.66 & 18.15 \\
\hline ship & 0.88 & 0.72 & 0.70 & 29.38 & 24.36 & 20.40 \\
\hline Lift & 0.83 & 0.65 & 0.70 & 28.74 & 24.45 & 25.84 \\
\hline
\end{tabular}

\section{CONCLUSIONS}

In the ROF model and Tikhonov model smaller value of regularization parameter $\lambda$ results in efficient noise removal, but lacks in preserving sharp edges. Higher value of $\lambda$ preserves fine details very well in the processed image. The ROF model for $\lambda=0.2$ performs very excellent in removing noise as well as preserving edges. Finding the appropriate value for Lagrange parameter to achieve effective denoising is done in an ad hoc manner. The ROF model outperforms in preserving edges because the diffusion exactly takes place along the edges. For the different values of parameter $\lambda$ $(0.003,0.01,0.1$ and 0.2$)$ the PSNR and SSIM remains almost same for TV-L1 model. In Tikhonov model strong image smoothing takes place compare to ROF model and TV-L1 model.

\section{REFERENCES}

[1] V. A. A. Tikhonov,"Solution of ill-posed problems", Winston, Washington, DC, 1977.

[2] I.Rudin, S. Osher, and E. Fatemi, "Nonlinear total variation based noise removal algorithms", Physica D: NonlinearPhenomena, 60(1):259-268, 1992.

[3] Beck and Teboulle, "Fast Gradient-based Algorithms for constrained Total Variation Image Denoisingand Deblurring Problems", IEEE Transcations on Image Processing, Vol. 18, No. 11, Nov. 2009
[4] Junfeng Yang, Yin Zhang and Wotao Yin," Efficient TV-L1 Algorithm for Deblurring Multichannel Images corrupted by impulse noise", SIAM Journal of. Scientific.Computing,Vol. 31, No. 4, pp. 2842-2865, 2009.

[5] Christian Clason, Bangti Jin and Karl Kunisch, "A Duality-based Splitting Method for $\ell-1-T V$ Image Restoration with Automatic Regularization Parameter Choice", SIAM Journal of Scientic. Computing, Vol. 32, No. 3, pp. 1484-1505, 2010.

[6] C. Clason. " $\ell-\infty$ fitting for inverse problems with uniform noise Inverse Problems", 28(10):104007, 2012.

[7] Haijuan Hu, Jacques Froment, "Nonlocal Total Variation for Image Denoising", Photonics and Optoelectronics (SOPO), 2012, May 2012, China. pp.1-4, 2012.

[8] Yan Jiang and Shu-Ling Zhang, "ImageDenoising Based on the Modied ROF Model", The authors - Published by Atlantis Press, 2013.

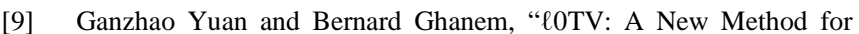
Image Restoration in the Presence of Impulse Noise", Computer Vision Pattern Recognition Conference 2015.

[10] Y. Wang, J. Yang, W. Yin, and Y. Zhang."A new alternating minimization algorithm for total variation image reconstruction", SIAM Journal on Imaging Sciences (SIIMS), 1(3):248-272, 2008.

[11] M. Yan. "Restoration of images corrupted by impulse noise and mixed gaussian impulse noise using blind inpainting", SIAM Journal on Imaging Sciences(SIIMS), 6(3):1227-1245, 2013.

[12] Li Guo, Weilong Chen, Yu Liao, Honghua Liao and JunLi,” An Edge-Preserved Image Denoising Based on Local Adaptive Regularisation", Journal of Sensors, Volume 2016 (2016).

[13] Xue-Cheng Tai and Chang-Hui Yao, "Image Segmentation by Piecewise Constant Mumford-Shah model without Estimating the Constants", Journal of Computational Math, No.3, 435-443, 2006.

[14] Qiangqiang Yuan, Liangpei Zhang and HuanfengShen, "Hyperspectral Image Denoising Employing a Spectral-Spatial Adaptive Total Variation Model", IEEE Transcations on Geoscience and Remote Sensing, 2012.

[15] Curtis R vogel and Mary E Oman, "Fast Robust Total Variationbased reconstruction of noisy, blurred images", IEEE Transcations on Image Processing, Vol. 7, Jun 1998.

[16] L. Xu, C. Lu, Y. Xu, and J. Jia. "Image smoothing via 10 gradient minimization",.ACM Transactions on Graphics, (TOG), 30(6):174, 2011.

[17] S.Karthik, Hemanth VK, K.P. Soman, V.Balaji, Sachin Kumar S, M. Sabarimalai Manikandan, "Directional Total Variation Filtering Based Image Denoising Method", International Journal of Computer Science Issues, Vol. 9, Issue 2, No 1, March 2012, ISSN (Online): 1694-0814

[18] Manya V. Afonso , João M.R. Sanches, “ A total variation recursive space-variant filter for image denoising”, ELSEVIER Digital Signal Processing, 2015.

[19] Yao Zhao et al. , “ Adaptive Total Variation Regularization Based SAR Image Despeckling and Despeckling Evaluation Index “, IEEE Transcations on Geoscience and Remote Sensing, VOL. 53, NO. 5, MAY 2015 .

[20] Kui Liu et al., "Hybrid regularizers-based adaptive anisotropic diffusion for image denoising", Journal SpringerPlus, 2016.

[21] Mazlinda Ibrahim, Ke Chen and Carlos Brito-Loeza, "A novel variational model for image registration using Gaussian curvature", Mathematics Numerical Analysis, April 2015. 